\title{
Kybernetika
}

\section{Mahmoud Gad}

Adaptive thresholding technique for solving optimization problems on attainable sets of (max, min)-linear systems

Kybernetika, Vol. 54 (2018), No. 2, 400-412

Persistent URL: http://dml.cz/dmlcz/147202

\section{Terms of use:}

(C) Institute of Information Theory and Automation AS CR, 2018

Institute of Mathematics of the Czech Academy of Sciences provides access to digitized documents strictly for personal use. Each copy of any part of this document must contain these Terms of use.

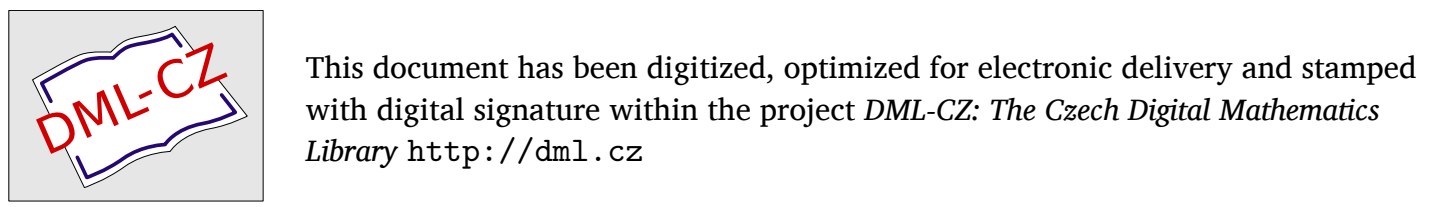




\title{
ADAPTIVE THRESHOLDING TECHNIQUE FOR SOLVING OPTIMIZATION PROBLEMS ON ATTAINABLE SETS OF (MAX, MIN)-LINEAR SYSTEMS
}

\author{
Mahmoud GAD
}

This article develops a parametric method depend on threshold technique for solving some optimization problems on attainable sets of so called ( $\max , \min )$-separable linear systems. The concept of attainable set for ( $\max , \min$ )-separable linear equation systems will be introduced. Properties of the attainable sets will be studied in detail. The (max, min) - separable linear equation systems, in which the function of unknown variable occur only on one side, will be consider.

The main idea of the proposed algorithm is that we will begin the calculations with the maximum element and we will try to decrease the value of the objective function of our problem, by decreasing its components in such a way that they stay within attainable set. Optimization problem consisting in finding the nearest point of an attainable set to a given fixed point will be considered. An algorithm for solving the optimization problem will be proposed. Motivational example from the area of operations research, which shows possible applications of the optimization problem solved in this paper, will be given. Numerical example illustrating the proposed algorithm is included.

Keywords: attainable sets, adaptive thresholding technique, (max;min)-separable equations

Classification: 90C47, 90C31

\section{INTRODUCTION}

Problems on algebraic structures, in which pairs of operations $(\max ,+)$ or $(\max , \min )$ replace addition and multiplication of the classical linear algebra have appeared in the literature approximately since the sixties of the last century (see e.g. [1]). In this paper we will study so called attainable sets of such systems, i.e. the sets of righthand sides, for which there exists a solution of the given system. Let us note that problems, the original formulation of which has no solution were called sometimes in the literature incorrectly posed problems ( see e.g. 3). Such problems are neither linear nor convex in usual algebraic sense. Such problems for $(\max ,+)$-linear equation system were considered using a different approach in (see e.g. [2, 9]). Problems for (max, min)linear equation and inequality system were considered using a different approach in (see

DOI: $10.14736 /$ kyb-2018-2-0400 
e.g. [4, 5, 7]). Our purpose in this paper is to present an approach to incorrectly posed (max, min)-separable equation systems, which depend on Adaptive Thresholding Technique.

Let us introduce the following notations: $I=\{1,2, \ldots, m\}, J=\{1,2, \ldots, n\}$. Let $A$ be a matrix with finite elements $a_{i j} \in R=(-\infty,+\infty), \forall \quad i \in I, \quad j \in J, \quad$ let $\alpha \wedge \beta \equiv \min (\alpha, \beta)$ for any $\alpha, \beta \in R$. Vector $A \otimes x \in R^{m}$ for $x=\left(x_{1}, \ldots, x_{n}\right)^{T} \in R^{n}$ will be defined as follows:

$$
(A \otimes x)_{i} \equiv \max _{j \in J}\left(a_{i j} \wedge x_{j}\right) \quad \forall i \in I .
$$

The system of (max, min)-separable equations with right-hand side $b \in R^{m}$ is an equation system of the form

$$
A \otimes x=b .
$$

The set of all solutions of the system will be denoted $M(b)$, (i. e. $M(b)=\left\{x \in R^{n} ; A \otimes\right.$ $x=b\}$.)

Definition 1.1. Set

$$
R(A) \equiv\left\{b \in R^{m} ; \exists x \in R^{n} \text { such that } A \otimes x=b\right\}
$$

is called attainable set of matrix $A$.

In what follows we will solve the following optimization problem:

Problem I. Minimize

$$
\|b-\hat{b}\|=\max _{i \in I}\left|b_{i}-\hat{b}_{i}\right|
$$

subject to

$$
b \in R(A) .
$$

The optimal solution of Problem I will be denoted $b^{o p t}$. Let us note that if $\hat{b} \in R(A)$, it is evidently $b^{o p t}=\hat{b}$. Therefore we will assume in what follows that $\hat{b} \notin R(A)$. Before investigating properties of attainable sets and analysis of problem solution, we will bring an example, which shows one possible application, which leads to solving the system given above.

Example 1.2. Let us assume that $m$ places $i \in I \equiv\{1,2, \ldots, m\}$ are connected with $n$ places $j \in J \equiv\{1,2, \ldots, n\}$ by roads with given capacities. The capacity of the road connecting place $i$ with place $j$ is equal to $a_{i j} \in R$. We have to extend for all $i \in I, j \in J$ the road between $i$ and $j$ by a road connecting $j$ with a terminal place $T$ and choose an appropriate capacity $x_{j}$ for this road. If a capacity $x_{j}$ is chosen, then the capacity of the road from $i$ to $T$ via $j$ is equal to $a_{i j} \wedge x_{j}=\min \left(a_{i j}, x_{j}\right)$. We require that the connection between places $i$ and $T$ is for at least one $j$ equal to a given number $b_{i} \in R$ and the chosen capacity $x_{j}$ lies in a given finite interval i. e. $x_{j} \in\left[\underline{x}_{j}, \bar{x}_{j}\right]$, where $\underline{x}_{j}, \bar{x}_{j} \in R$ are given finite numbers. Therefore feasible vectors of capacities $x=\left(x_{1}, x_{2}, \ldots, x_{n}\right)$ (i. e. the vectors, the components of which are capacities $x_{j}$ having the required properties) must satisfy an equation system of the form $A \otimes x=b$. 


\section{PROPERTIES OF ATTAINABLE SETS AND ANALYSIS OF THE PROBLEM}

In this section we will study in more detail some properties of attainable sets and analyze Problem I. The properties are formulated as the following three lemmas.

Lemma 2.1. Set $R(A)$ has the maximum element, i. e. an element $b^{\max } \in R(A)$ such that $b \leq b^{\max } \forall b \in R(A)$.

Proof. Let $\alpha_{i}=\max _{j \in J} a_{i j} \forall i \in I$. Let $x \in R^{n}$ be arbitrarily chosen. Then $a_{i j} \wedge x_{j} \leq a_{i j}$ for all $i \in I, j \in J$. Therefore for any $i \in I$ we obtain that

$$
\max _{j \in J}\left(a_{i j} \wedge x_{j}\right) \leq \max _{j \in J} a_{i j}=\alpha_{i} .
$$

Therefore if we set $b_{i}^{\max }=\alpha_{i} \forall i \in I$, then $b^{\max } \in R(A)$, since e.g. if $\hat{x} \in R^{n}$ and $\hat{x}_{j} \geq \max _{i \in I} \alpha_{i}$ we have $\max _{j \in J}\left(a_{i j} \wedge \hat{x}_{j}\right)=\alpha_{i}=b_{i}^{\max }$. For an arbitrary $b \in R(A)$ there exists $x \in R^{n}$ such that $b=A \otimes x \leq A \otimes \hat{x}=b^{\max }$, so that $b^{\text {max }}$ is the maximum element of $R(A)$, this ends the proof.

Lemma 2.2. (Zimmermann [8]) Let $b \in R^{m}, I_{j}^{>}=\left\{i \in I ; a_{i j}>b_{i}\right\} \quad \forall j \in J$. Let $M(b)=\left\{x \in R^{n} ; A \otimes x=b\right\}$ be nonempty. Let vector $x(b) \in R^{n}$ be defined as follows:

$$
x_{j}(b)=\min _{i \in I_{j}^{>}} b_{i} \forall j \in J \text { if } I_{j}^{>} \neq \emptyset .
$$

We set the minimum equal to infinity if $I_{j}^{>}=\emptyset$. Then $x(b)$ is the maximum element of set $M(b)$.

Proof. Let us note that if $x \in M(b)$, then it must be $a_{i j} \wedge x_{j} \leq b_{i}$ for all $i \in I, j \in J$. Therefore it must be $x \leq x(b) \forall x \in M(b)$ so that $x(b)$ is the upper bound for elements of $M(b)$. It remains to prove that if set $M(b)$ is nonempty it must be $x(b) \in M(b)$. Let us set

$$
S_{j}\left(x_{j}\right) \equiv\left\{k \in I ; a_{k j} \wedge x_{j}=b_{k}\right\} \quad \forall j \in J .
$$

If $I_{j}^{>} \neq \emptyset$, then

$$
S_{j}\left(x_{j}(b)\right)=\left\{k \in I ; x_{j}(b)=b_{k}=\min _{i \in I_{j}^{>}}\left(b_{i}\right)\right\} .
$$

If $I_{j}^{>}=\emptyset$, then $x_{j}(b)=\infty$ and $S_{j}\left(x_{j}(b)\right)=\left\{k \in I ; a_{k j}=b_{k}\right\}$. We will show further that

$$
x(b) \in M(b) \Longleftrightarrow \bigcup_{j \in J} S_{j}\left(x_{j}(b)\right)=I
$$

Really if $\bigcup_{j \in J} S_{j}\left(x_{j}(b)\right)=I$ and $p \in I$ is arbitrary, then there exists index $j(p) \in J$ such that $p \in S_{j(p)}\left(x_{j(p)}(b)\right)$ and therefore $a_{p j} \wedge x_{j}(b) \leq b_{p}$ for all $j \in J$ and $a_{p j(p)} \wedge x_{j(p)}(b)=$ $b_{p}$ so that $\max _{j \in J}\left(a_{p j} \wedge x_{j}(b)\right)=b_{p}$. Since $p$ was arbitrary, we obtain that $x(b) \in M(b)$. To prove the opposite implication let us assume that $\bigcup_{j \in J} S_{j}\left(x_{j}(b)\right) \neq I$ so that there exists index $i_{0} \in I$ such that $i_{0} \notin \bigcup_{j \in J} S_{j}\left(x_{j}(b)\right)$ and therefore $a_{i_{0} j} \wedge x_{j}(b) \neq b_{i_{0}} \quad \forall j \in J$ and therefore $\max _{j \in J}\left(a_{i_{0} j} \wedge x_{j}(b)\right) \neq b_{i_{0}}$ and thus $x(b) \notin M(b)$. 
Let us note that if $x_{j} \leq x_{j}(b)$ for any $j \in J$, then $S_{j}\left(x_{j}\right) \subseteq S_{j}\left(x_{j}(b)\right)$. Therefore if $\bigcup_{j \in J} S_{j}\left(x_{j}(b)\right) \subset I$, then for any $x \leq x(b)$ we have

$$
\bigcup_{j \in J} S_{j}\left(x_{j}\right) \subseteq \bigcup_{j \in J} S_{j}\left(x_{j}(b)\right) \subset I
$$

and thus $M(b)=\emptyset$, since all elements of $M(b)$ must satisfy the inequality $x \leq x(b)$. It follows that

$$
M(b) \neq \emptyset \quad \Longleftrightarrow \quad x(b) \in M(b) .
$$

In other words if $M(b) \neq \emptyset$, then $x(b) \in M(b)$ and $x \leq x(b)$ for all $x \in M(b)$, so that $x(b)$ in the maximum element of $M(b)$, what was to be proved.

Lemma 2.3. Let $b^{\max }$ be the maximum element of $R(A), \hat{b} \in R^{m}$ such that $\hat{b} \geq b^{\max }$, let $b^{\text {opt }}$ be the optimal solution of Problem I. Then $b^{\text {opt }}=b^{\text {max }}$.

Proof. Since $b^{\max }$ is the maximum element of $R(A)$, so $b \leq b^{\max }$ for any an arbitrary element $b \in R(A)$, then $b_{p} \leq b_{p}^{\max }$ for all $p \in I$. Since $\hat{b} \geq b^{\max }$, Then $\left|b_{p}^{\max }-\hat{b}_{p}\right| \leq$ $\left|b_{p}-\hat{b}_{p}\right| \quad$ for all $p \in I \quad$ it follows that $\left\|b^{\max }-\hat{b}\right\| \leq\|b-\hat{b}\|$ for all $b \in R(A)$, which implies that $b^{o p t}=b^{\max }$.

In the next section, we will propose an algorithm for solving Problem I.

\section{THE THRESHOLD ALGORITHM}

Let us introduce the following notations, we assume that $b \in R(A), \hat{b} \notin R(A), i \in I$ :

$$
H^{+}(b)=\left\{i \in I ; b_{i}>\hat{b}_{i}\right\}, H^{-}(b)=I \backslash H^{+}(b),
$$

Let us define $\hat{x}(b) \in R^{n}$ as follows:

$$
\hat{x}_{j}(b)= \begin{cases}\min _{i \in I_{j}^{>}} b_{i}, & \text { if } I_{j}^{>} \neq \emptyset, \\ \max _{i \in I_{j}^{-}} b_{i}, & \text { if } I_{j}^{=} \neq \emptyset \text { and } I_{j}^{>}=\emptyset, \\ \infty & \text { otherwise, }\end{cases}
$$

where $I_{j}^{>}=\left\{i \in I ; a_{i j}>b_{i}\right\} \forall j \in J, I_{j}^{=}=\left\{i \in I ; a_{i j}=b_{i}\right\} \forall j \in J$ such that $I_{j}^{>}=\emptyset$.

Let us set further

$$
\begin{gathered}
G_{i}(b)=\left\{h \in J ; a_{i h} \wedge \hat{x}_{h}(b)=\max _{j \in J}\left(a_{i j} \wedge \hat{x}_{j}(b)\right)\right\}, \\
P(b)=\left\{i \in I ; a_{i h} \wedge \hat{x}_{h}(b)=\hat{x}_{h}(b) \forall h \in G_{i}(b)\right\} .
\end{gathered}
$$

We will call terms $a_{i h} \wedge \hat{x}_{h}(b)$ in the definition of $G_{i}(b)$ "active terms of $G_{i}(b)$ ". If $i_{0} \in P(b)$, then all active terms of $G_{i_{0}}(b)$ are equal to $\hat{x}_{h}(b)$, or in other words $a_{i_{0} h} \wedge$ $\hat{x}_{h}(b)=\hat{x}_{h}(b)$, which means that $a_{i_{0} h} \geq \hat{x}_{h}(b) \forall h \in G_{i_{0}}(b)$. Let us set

$$
F^{+}(b)=\left\{k \in H^{+}(b) \cap P(b) ;\left|b_{k}-\hat{b}_{k}\right|=\max _{i \in I}\left|b_{i}-\hat{b}_{i}\right|\right\},
$$




$$
F^{-}(b)=\left\{k \in H^{-}(b) \cap P(b) ;\left|b_{k}-\hat{b}_{k}\right|=\max _{i \in I}\left|b_{i}-\hat{b}_{i}\right|\right\} .
$$

The main idea of the proposed algorithm is that we will begin the calculations with the maximum element $b^{\max }$ and will try to decrease the value of the objective function of Problem I, by decreasing components of $b$ in such a way that we stay within attainable set $R(A)$. We will assume $I_{j}^{>} \neq \emptyset$ for all $j \in J$ to avoid infinite components of $\hat{x}(b)$. Let

$$
\bar{G}(\bar{b}) \equiv \bigcup_{k \in F^{+}(\bar{b})} G_{k}(\bar{b}) .
$$

\section{Algorithm 3.1.}

0 Input $m, n, I, J, A, \hat{b}, b^{\max }, \bar{b}:=b^{\max }$

1 Determine $H^{+}(\bar{b}), H^{-}(\bar{b}), \hat{x}(\bar{b}), G_{i}(\bar{b}) \forall i \in I, P(\bar{b}), F^{+}(\bar{b}), F^{-}(\bar{b})$.

2 If $F^{-}(\bar{b}) \neq \emptyset$, go to 8 .

$3 \tilde{F}(\bar{b}):=\left\{i \in\left(I \backslash F^{+}(\bar{b})\right) \cap P(\bar{b}) ; G_{i}(\bar{b}) \subseteq \bigcup_{k \in\left(F^{+}(\bar{b}) \cap P(\bar{b})\right)} G_{k}(\bar{b})\right\}$.

$4 T(\bar{b}):=F^{+}(\bar{b}) \cup \tilde{F}(\bar{b})$; if $T(\bar{b})=\emptyset$, go to 8 .

5 set for $t \geq 0: b_{i}(t):=\bar{b}_{i}-t \quad \forall i \in T(\bar{b}), b_{i}(t):=\bar{b}_{i}$ otherwise.

6 Increase $t$ until a value $\tau>0$, for which for the first time one of the following events will occur:

(i) $b_{i}(\tau)=\hat{b}_{i}$ for some $i \in T(\bar{b})$;

(ii) $b_{i}(\tau)=\bar{b}_{p}$ for some $i \in T(\bar{b}), p \in I \backslash T(\bar{b})$;

(iii) $\left|b_{i}(\tau)-\hat{b}_{i}\right|=\max _{k \in(I \backslash T(\bar{b}))}\left|\bar{b}_{k}-\hat{b}_{k}\right|$ for some $i \in T(\bar{b})$. It may happen that $G_{p}(\bar{b}) \nsubseteq \bar{G}(\bar{b})$ so that $p \notin \tilde{F}(\bar{b})$, but $G_{p}(\bar{b}-t) \subseteq \bar{G}(\bar{b})$ so that $p \in \tilde{F}(\bar{b}-t) \cap P(\bar{b}-t)$;

(iv) $\left|b_{i}(\tau)-\hat{b}_{i}\right|=\left|b_{k}(\tau)-\hat{b}_{k}\right|$, where $i \in \hat{F}^{+}(\bar{b})$ and $k \in T(\bar{b}) \cap H^{-}(\bar{b})$.

(v) $P(\bar{b})$ may change, i. e. until for some $t=\tau$ may be $P(b(\tau)) \neq P(\bar{b}), \bar{b}_{i}-t=$ $\max _{j \in J \backslash G_{i}(\bar{b})} a_{i j}$ for some $i \in T(\bar{b})$.

Find $\tau$ by making use of Algorithm 3.2 .

7 Set $\bar{b}:=b(\tau)$, go to 1 .

8 Set $b^{o p t}:=\bar{b}$, STOP. 
Through this algorithm we begin the calculations with the maximum element $b^{\text {max }}$ and will try to decrease the value of the objective function of Problem I. So we determine the indices of the components of $b$ have to be decreased in such a way that we stay within attainable set $R(A)$. as follows:

In step 2

Set $F^{-}(\bar{b})$ contains indices, for which $\hat{b}_{k}-\bar{b}_{k}=\|\bar{b}-\hat{b}\|$. Since $\bar{b}_{k}<\hat{b}_{k}$ and $\bar{b}_{k}$ must be decreased (it is the maximum element of $R(A)$ ), we can only increase the value of the objective function and therefore we stop the calculations with $\bar{b}=b^{\max }$.

In step 3

Set $\tilde{F}(\bar{b})$ contains indices of $\bar{b}$, which are not active at point $\bar{b}$ in the objective function value $\|\bar{b}-\hat{b}\|$ and their decrease is induced by the decrease of active components of $\bar{b}$ (i. e. variables $\bar{b}_{k}$, which determine the value $\|\bar{b}-\hat{b}\|$ ).

In step 4

Set $T(\bar{b})$ contains indices of components $\bar{b}_{s}$, which will be decreased if we want to decrease the value $\|\bar{b}-\hat{b}\|$, i. e. $\bar{b}_{k}, k \in F^{+}(\bar{b})$, which must be decreased and variables $\bar{b}_{r}, r \in \tilde{F}(\bar{b})$, the decrease of which is induced (or forced) by the decrease of the components of $\bar{b}$, which take an active part in determining the value $\|\bar{b}-\hat{b}\|$.

In step 5

The step carries out the uniform contemporary parametric decrease of variables $\bar{b}_{s}, s \in T(\bar{b})$.

In step 6

This step finds a value $\tau>0$ of parameter $t$, at which for the first time at least one of the threshold index sets $H^{+}(\bar{b}), H^{-}(\overline{(b}), G_{i}(\bar{b}), i \in I, F^{+}(\bar{b}), F^{-}(\bar{b}), P(\bar{b})$ changes. Note that all these threshold index sets remain unchanged if $b=b(t), t \in[0, \tau]$ and if $t>\tau$. If $t=\tau$, then at least one of the threshold sets changes. Points (i) $-(\mathrm{v})$ determine the situations, at which the threshold sets change. The corresponding values of parameter $t$,at which these changes take place will be computed in Algorithm 3.2

In what follows we will bring an Algorithm 3.2 for determining the parameter value $\tau$ from step 6 of Algorithm 3.1 . For this purpose we will introduce the following simplifying notations:

$$
\begin{gathered}
\alpha(\bar{b}) \equiv\|\bar{b}-\hat{b}\|, \\
\beta(\bar{b}) \equiv \max _{i \in I \backslash T(\bar{b})}\left|\bar{b}_{i}-\hat{b}_{i}\right| .
\end{gathered}
$$

Let us recall that $b_{i}(t)=\bar{b}_{i}-t \forall i \in T(\bar{b}), b_{i}(t)=\bar{b}_{i} \forall i \in I \backslash T(\bar{b})$. We have then:

$\mid \begin{aligned} & b_{i}(t)-\hat{b}_{i} \mid=b_{i}(t)-\hat{b}_{i}=\bar{b}_{i}-t-\hat{b}_{i} \quad \forall i \in F^{+}(\bar{b}) \\ & b_{i}(t)-\hat{b}_{i} \mid=\hat{b}_{i}-b_{i}(t)=\hat{b}_{i}-\bar{b}_{i}+t \forall i \in H^{-}(\bar{b}) \cap T(\bar{b}) .\end{aligned}$

We will analyze in detail cases $(\mathrm{i})$ - (v) from step 6 . 
Case (i) - changes $H^{+}(\bar{b})$. We have for $i \in T(\bar{b})$ $b_{i}(t)=\hat{b}_{i}$ if $t=\tau_{i}^{(1)} \equiv\left(\bar{b}_{i}-\hat{b}_{i}\right)$. Case (i) takes place for the first time if

$$
t=\tau^{(1)} \equiv \min _{i \in T(\bar{b})} \tau_{i}^{(1)}
$$

Case (ii) - changes $T(\bar{b})$. We have $b_{i}(t)=\bar{b}_{p}$ for some $i \in T(\bar{b}), p \in I \backslash T(\bar{b})$ if $t=\tau_{i p}^{(2)} \equiv \bar{b}_{i}-\bar{b}_{p}$. Case (ii) takes place for the first time if

$$
t=\tau^{(2)}=\min _{i \in T(\bar{b}), p \notin T(\bar{b})} \tau_{i p}^{(2)} .
$$

Case (iii) - changes $F^{+}(\bar{b})$. We have $\|b(t)-\hat{b}\|=\alpha(\bar{b})-t$, so that $\|b(t)-\hat{b}\|=$ $\beta(\bar{b}) \quad$ if

$$
t=\tau^{(3)} \equiv(\alpha(\bar{b})-\beta(\bar{b}))
$$

Case (iv) - changes $F^{-}(\bar{b})$. We have for $k \in T(\bar{b}) \cap H^{-}(\bar{b})$ the equality $\left|b_{k}(t)-\hat{b}_{k}\right|=$ $\hat{b}_{k}-\bar{b}_{k}+t$ so that $\|b(t)-\hat{b}\|=\left|b_{k}(t)-\hat{b}_{k}\right|$ if $\alpha(\bar{b})-t=\left(\hat{b}_{k}-\bar{b}_{k}+t\right)$, i. e. if $t=\tau_{k}^{(4)} \equiv$ $\left(\alpha(\bar{b})-\hat{b}_{k}+\bar{b}_{k}\right) / 2$. Case (iv) takes place for the first time if

$$
t=\tau^{(4)} \equiv \min _{k \in\left(T(\bar{b}) \cap H^{-}(\bar{b})\right)} \tau_{k}^{(4)} .
$$

Case (v) - changes $\bar{G}(\bar{b})$.

$$
\bar{b}_{i}-t=\max _{j \in J \backslash G_{i}(\bar{b})}\left(a_{i j}\right) \text {, for some } i \in T(\bar{b})
$$

i. e.

$$
t=\tau_{i}^{5}=\bar{b}_{i}-\max _{j \in J \backslash G_{i}(\bar{b})}\left(a_{i j}\right)
$$

for some $i \in T(\bar{b})$.

We set then $\tau^{(5)}=\min _{i \in T(\bar{b})} \tau_{i}^{(5)}$.

One of the Cases (i) - (v) takes place for the first time if

$$
t=\tau \equiv \min _{1 \leq k \leq 5} \tau^{(k)}
$$

Value $\tau>0$ will be inserted in step 6 of algorithm 3.1 . We will summarize these considerations in the following 
Algorithm 3.2.

1 Input $\hat{b}, \bar{b}, \alpha(\bar{b}), \beta(\bar{b})$.

$2 \tau_{i}^{(1)} \equiv\left(\bar{b}_{i}-\hat{b}_{i}\right) \quad \forall i \in T(\bar{b})$,

$$
\tau^{(1)}:=\min _{i \in T(\bar{b})} \tau_{i}^{(1)}
$$

$3 \tau_{i p}^{(2)} \equiv \bar{b}_{i}-\bar{b}_{p}, \forall i \in T(\bar{b}), p \notin T(\bar{b})$,

$$
t=\tau^{(2)}=\min _{i \in T(\bar{b}), p \notin T(\bar{b})} \tau_{i p}^{(2)} .
$$

$4 \tau^{(3)} \equiv(\alpha(\bar{b})-\beta(\bar{b}))$.

$5 \tau_{k}^{(4)} \equiv\left(\alpha(\bar{b})-\hat{b}_{k}+\bar{b}_{k}\right) / 2 \quad \forall k \in T(\bar{b}) \cap H^{-}(\bar{b})$,

$$
\tau^{(4)} \equiv \min _{k \in\left(T(\bar{b}) \cap H^{-}(\bar{b})\right)} \tau_{k}^{(4)}
$$

6

$$
\tau^{(5)} \equiv \min _{i \in T(\bar{b})}\left(\bar{b}_{i}-\max _{j \in J \backslash G_{i}(\bar{b})}\left(a_{i j}\right)\right)
$$

7

$$
\tau \equiv \min _{1 \leq k \leq 5} \tau^{(k)}
$$

Remark 3.3. In the theory presented above we assumed that the entries of $A$ and $b$ are real numbers. Note that the obtained results can be extended to cases, where the entries are assumed to be e.g. rational numbers, integers or even given finite sets of numbers.

Remark 3.4. In case that the entries of Problem I are rational or integer numbers, the proposed algorithm has polynomial number of steps. Namely, each iteration of the algorithm is connected with at least one change of a threshold index set connected with reaching at least one threshold value generated by elements of $A$ or components of $\hat{b}$. Each variable $x_{j}$ can be stopped $m-1$ times by $m-1$ elements of $A$ in the $j$ th column when $\hat{x}_{j}(b(t))>a_{i j}$ and again set into movement when at a further current $x_{j}(\bar{b}(t))$ the equality $\hat{x}_{j}(\bar{b})=a_{i j}$ is reached (i.e. $P(\bar{b})$ can change with the current decreased $b(t)$ $m-1$ times. Besides $b_{k}(t), k \in \tilde{F}_{k}(b(t))$ can be stopped $m-1$ times when $\tilde{F}(b(t))$ changes and similarly can change $F^{+}(b(t))$ if an active $b_{k}(t)$ reaches value $\hat{b}_{k}$. This makes together $O(m n)$ steps before a final step 8 . The computation of $\tau$ in Algorithm 3.2 needs $O(m)$ resp. $O(n)$ steps.

Remark 3.5. An alternative method for solving problems similar to Problem I was suggested in [6]. Neither the method presented here nor the method in [6] can be preferred in general. The effectiveness of the methods depends on the structure of examples solved. If e.g. the number of elements of $F^{+}(\bar{b})$ is low, the method presented here seems to be more effective than the method in [6]. 


\section{NUMERICAL EXAMPLES}

We illustrate the theoretical result by the next numerical example,

\section{Example 4.1.}

Let $m=n=3, \hat{b}=(8,8,8)^{T}$,

$$
A=\left(\begin{array}{lll}
3 & 1 & 5 \\
4 & 4 & 6 \\
7 & 7 & 3
\end{array}\right)
$$

In this case $b^{\max }=(5,6,7)^{T} \leq \hat{b}=(8,8,8)^{T}$. Taking into account Lemma 2.3. we obtain that the optimal solution of Problem $\mathrm{I}$ is equal to $b^{\max }$. The optimal value of the objective function of Problem I is therefore $\left\|b^{\max }-\hat{b}\right\|=\max (3,2,1)=3$.

Let us solve the same problem using the threshold algorithm from the preceding section. The iterations of this algorithm will be the following:

$1 m=n=3, I, J, A, \hat{b}, \bar{b}=b^{\max }=(5,6,7)^{T} ;$

\section{Iteration 1}

$2 \bar{b}=(5,6,7), H^{+}(\bar{b})=I, H^{-}(\bar{b})=\emptyset, \hat{x}(\bar{b})=(7,7,6)^{T}, G_{1}(\bar{b})=\{3\}, G_{2}(\bar{b})=$ $\{3\}, G_{3}(\bar{b})=\{1,2\}, P(\bar{b})=\{2,3\}, F^{+}(\bar{b})=\{3\}$;

$3 F^{-}(\bar{b})=\emptyset$;

$4 \tilde{F}(\bar{b})=\emptyset$;

$5 T(\bar{b})=\{3\} ;$

$6 b(t)=(5,6,7-t)^{T}$

$7 \tau=\min (7,1,1,+\infty)=1$;

$8 \bar{b}:=b(\tau)=(5,6,6)^{T}$.

\section{Iteration 2}

$2 \bar{b}=(5,6,6), H^{+}(\bar{b})=I, H^{-}(\bar{b})=\emptyset, \hat{x}(\bar{b})=(6,6,6), \max _{j \in J}\left(a_{1 j} \wedge x_{j}(\bar{b})\right)=a_{13}=5$, so that $G_{1}(\bar{b})=\{3\}, \max _{j \in J}\left(a_{2 j} \wedge x_{j}(\bar{b})\right)=x_{3}(\bar{b})=6, G_{2}(\bar{b})=\{3\}, \max _{j \in J}\left(a_{3 j} \wedge\right.$ $\left.x_{j}(\bar{b})\right)=x_{1}(\bar{b})=x_{2}(\bar{b})=7$ so that $G_{3}(\bar{b})=\{1,2\}$, futher we have $P(\bar{b})=\{2,3\}$ so that $F^{+}(\bar{b})=\{2,3\}, F^{-}(\bar{b})=\emptyset, ;$

$3 F^{-}(\bar{b})=\emptyset$;

$4 \tilde{F}(\bar{b})=\emptyset$;

$5 T(\bar{b})=\{2,3\} ;$

$6 b(t)=(5,6-t, 6-t)$;

$7 \tau=1$;

$8 \bar{b}=b(\tau)=(5,5,5)^{T}$; 


\section{Iteration 3}

$$
\begin{aligned}
& 2 \bar{b}=(5,5,5), H^{+}(\bar{b})=I, H^{-}(\bar{b}), \hat{x}(\bar{b})=(5,5,5), F^{+}(\bar{b})=\{1\} ; \\
& 3 F^{-}(\bar{b})=\emptyset ; \\
& 4 \tilde{F}(\bar{b})=\{2\} ; \\
& 5 T(\bar{b})=\{1,2\} ; \\
& 6 b(t)=(5-t, 5-t, 5) ; \\
& 7 \tau=1 ; \\
& 8 \bar{b}=b(\tau)=(4,4,5)^{T} ;
\end{aligned}
$$

\section{Iteration 4}

$2 \bar{b}=(4,4,5)^{T} H^{+}(\bar{b})=I, H^{-}(\bar{b})=\emptyset, \hat{x}(\bar{b})=(5,5,4), G_{1}(\bar{b})=\{3\}, G_{2}(\bar{b})=$ $\{1,2,3\}, G_{3}(\bar{b})=\{1,2\}, P(\bar{b})=\{1,3\}, F^{+}(\bar{b})=\{1,3\}$;

$$
\begin{aligned}
& 3 F^{-}(\bar{b})=\emptyset ; \\
& 4 \tilde{F}(\bar{b})=\emptyset ; \\
& 5 T(\bar{b})=\{1,3\} ; \\
& 6 \quad b(t)=(4-t, 4,5-t) ; \\
& 7 \tau=1 ; \\
& 8 \bar{b}=b(\tau)=(3,4,4)^{T} ;
\end{aligned}
$$

\section{Iteration 5}

$$
\begin{aligned}
& 2 \bar{b}=(3,4,4), H^{+}(\bar{b})=I, H^{-}(\bar{b})=\emptyset, \hat{x}(\bar{b})=(4,4,3), P(\bar{b})=\{2,3\}, F^{+}(\bar{b})=\{2,3\} ; \\
& 3 F^{-}(\bar{b})=\emptyset ; \\
& 4 \tilde{F}(\bar{b})=\emptyset \\
& 5 \text { T }(\bar{b})=\{2,3\} ; \\
& 6 \text { b } b(t)=(3,4-t, 4-t) ; \\
& 7 \tau=1 ; \\
& 8 \bar{b}:=b(\tau)=(3,3,3) ;
\end{aligned}
$$




\section{Iteration 6}

$2 \bar{b}=(3,3,3), H^{+}(\bar{b})=I, H^{-}(\bar{b})=\emptyset, \hat{x}(\bar{b})=(3,3,3), P(\bar{b})=(1,2,3), F^{+}(\bar{b})=\{1\}$;

$3 F^{-}(\bar{b})=\emptyset$;

$4 \tilde{F}(\bar{b})=\emptyset$;

$5 T(\bar{b})=\{1\} ;$

$6 b(t)=(3-t, 3,3)$;

$7 \tau=1$;

$8 \bar{b} ;=b(\tau)=(2,3,3)$;

\section{Iteration 7.}

$2 \bar{b}=(2,3,3), H^{+}(\bar{b})=I, H^{-}(\bar{b})=\emptyset, \hat{x}(\bar{b})=(2,3,2), \quad P(\bar{b})=\{1,2,3\}, F^{+}(\bar{b})=$ $\{1,2,3\}$;

$3 F^{-} \bar{b}=\emptyset$;

$4 \tilde{F}(\bar{b})=\emptyset$;

$5 T(\bar{b})=\{1\} ;$

$6 b(t)=(2-t, 3-t, 3-t)$;

$7 \tau=1$;

$8 \bar{b}:=b(\tau)=(1,2,2)$;

\section{Iteration 8}

$2 \bar{b}=(1,2,2), H^{+}(\bar{b})=I, H^{-}(\bar{b})=\emptyset, \hat{x}(\bar{b})=(1,2,1), G_{1}(\bar{b})=\{1,2,3\}, G_{2}(\bar{b})=$ $\{2\}, G_{3}(\bar{b})=\{2\}, P(\bar{b})=\{1,2,3\}, F^{+}(\bar{b})=\{2,3\}$;

$3 F^{-}(\bar{b})=\emptyset$;

$4 \tilde{F}(\bar{b})=\emptyset$;

$5 T(\bar{b})=\{1,2,3\} ;$

$6 b(t)=(1,2-t, 2-t)$;

$7 \tau=\tau^{(5)}=1$;

$8 \bar{b}:=b(\tau)=(1,1,1)$; 


\section{Iteration 9}

$2 \bar{b}:=(1,1,1), H^{+}(\bar{b})=\{1\}, H^{-}(\bar{b})=\{2,3\}, \hat{x}(\bar{b})=(1,1,1), G_{i}(\bar{b})=I \forall i \in$ $I, P(\bar{b})=I, F^{+}(\bar{b})=\{1\}$;

$3 F^{-}(\bar{b})=\emptyset$;

$4 \tilde{F}(\bar{b})=\{2,3\}$;

$5 T(\bar{b})=\{1,2,3\}$;

$6 b(t)=(1-t, 1-t, 1-t)$;

$7 \tau=\tau^{(4)}=1$;

$8 \bar{b}:=b(\tau)=(1 / 2,1 / 2,1 / 2)$;

\section{Iteration 10}

$2 \bar{b}=(1 / 2,1 / 2,1 / 2), H^{+}(\bar{b})=\{1\}, H^{-}(\bar{b})=\{2,3\}, \hat{x}(\bar{b})=(1 / 2,1 / 2,1 / 2)$,

$F^{+}(\bar{b})=\{1\}$

$3 F^{-}(\bar{b})=\{2,3\} \neq \emptyset$;

$9 b^{\text {opt }}:=\bar{b}=(1 / 2,1 / 2,1 / 2)$, STOP.

\section{CONCLUSIONS}

In this paper the idea that arises in connection with practical applications, which are described by (max, min)-separable linear equation systems, is introduced. The problem what to do if the given ( $\max , \min$ )-separable linear equation systems has no feasible solution is analysed. We have to modify the original system (i.e. to modify its input coefficients) in such a way that the new problem has a solution. In this situation it is natural trying to modify the problems in such a way that the original goals of the given system ( e.g. bounds on costs or arrival times) will be violated as little as possible. We introduced a technique through it we can modify the values on the right hand side of the systems until the system is solvable, and it will be violated as little as possible. Moreover, we will try to apply the technique introduced here for real life problems and introduce a new technique that allows modify the values of the left hand side coefficients in order the system has a feasible solution. Another possibility to make the research closer to practical requirements would be considering stochastic or interval input coefficients of the (max, min)-linear systems.

\section{ACKNOWLEDGMENT}

The research is supported by the Cultural Affairs and Missions Sector - Egyptian Ministry of Higher Education, Sohag University in Sohag - Egypt; Faculty of Informatics and Statistics, University of Economics, Prague; and Faculty of Mathematics and Physics - Charles University, Prague - Czech Republic. 
[1] P. Butkovič: Max-linear Systems: Theory and Algorithms. Springer Monographs in Mathematics and Springer-Verlag, London - Dodrecht - Heudelberg - New York 2010. DOI:10.1007/978-1-84996-299-5

[2] R. A. Cuninghame-Green: Minimax Algebra, Lecture Notes in Economics and Mathematical Systems. Springer-Verlag 166, Berlin 1979. DOI:10.1007/978-3-642-48708-8

[3] I. I. Eremin, V.D. Mazurov, and N. N. Astafev: Linear Inequalities in mathematical programming and pattern recognition. Ukr. Math. J. 40 (1988), 3, 243-251. Translated from Ukr. Mat. Zh. 40 (1988), 3, 288-297. DOI:10.1007/bf01061299

[4] M. Gad: Optimization problems under one-sided (max, min)-linear equality constraints. In: WDS'12 Proc. Contributed Papers Part I, 2012, pp.13-19. 21st Annual Student Conference, Week of Doctoral Students Charles University, Prague 2012.

[5] M. Gad: Optimization problems under two-sided (max, min)-linear inequalities constraints. Academic Coordination Centre J. 18 (2012), 4, 84-92. In: International Conference Presentation of Mathematics Conference ICPM'12, Liberec 2012.

[6] M. Gad, J. Jablonský, and K. Zimmermann: Incorrectly posed optimization problems under extremally linear equation constraints. In: Proc. 34th International Conference of Mathematical Methods in Economics MME 2016, Liberec 2016, pp. 231-236.

[7] M. Gavalec, M. Gad, and K. Zimmermann: Optimization problems under (max, min)linear equations and/or inequality constraints. J. Math. Sci. 193 (2013), 5, 645-658. Translated from Russian Journal Fundamentalnaya i Prikladnaya Matematika(Fundamental and Applied Mathematics) 17 (2012), 6, 3-21.

[8] K. Zimmernann: Extremální algebra (in Czech). Ekon. ústav ČSAV, Praha 1976.

[9] K. Zimmermann and M. Gad: Optimization problems under one-sided (max, + )-linear constraints. In: Conference Presentation of Mathematics ICPM'11, Liberec 2011, pp. 159 165.

Mahmoud Gad, University of Economics, Faculty of Informatics and Statistics, Department of Econometrics, W. Churchill Sq. 1938/4, 13067 Praha 3, Czech Republic and Sohag University, Faculty of Science, Sohag 82524. Egypt.

e-mail: Mahmoud_attya_or@yahoo.com 